
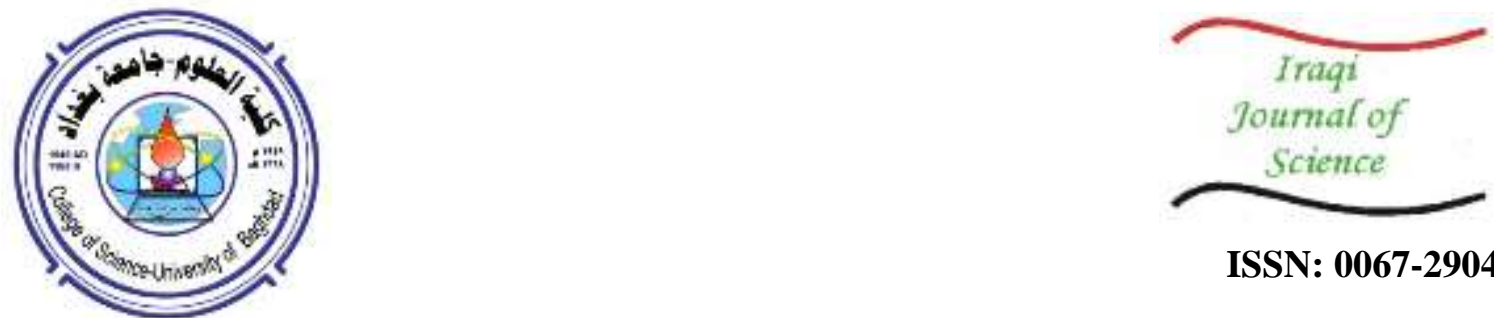

ISSN: 0067-2904

\title{
MHD Effect on Peristaltic Transport for Rabinowitsch Fluid through A Porous Medium in Cilia Channel
}

\author{
Saba S. Hasen, Ahmed M. Abdulhadi \\ Department of Mathematics, College of Science, University of Baghdad Baghdad, Iraq
}

Received: 5/9/2019 Accepted: 22/10/2019

\begin{abstract}
This paper is employed to discuss the effects of the magnetic field and heat transfer on the peristaltic flow of Rabinowitsch fluid through a porous medium in the cilia channel. The governing equations (mass, motion, and energy) are formulated and then the assumptions of long wavelength and low Reynold number are used for simplification. The velocity field, pressure gradient, temperature, and streamlines are obtained when the perturbation technique is applied to solve the nonlinear partial differential equations. The study shows that the velocity is decreased with increasing Hartmann number while it is decreased with increasing the porosity.
\end{abstract}

Keywords: Peristalsis flow, Rabinowitsch fluid, symmetric channel, porous medium, (MHD) magnetohydrodynamic field.

\section{التأثير المغناطيسي على الانتقال التمجي لمائع رابينوج خلال وسط مسامي في قناة مهابة}

$$
\begin{aligned}
& \text { صبا ستار حسن*"، احمد مولود عبد الهادي }
\end{aligned}
$$

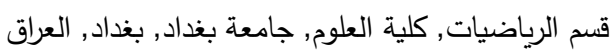

$$
\begin{aligned}
& \text { الخلاصة } \\
& \text { هذا البحث وظف لمناقثة تأثير المجال المغناطيسي ونقل الحرارة على التدفق التمعجي لسائل رابينوج من }
\end{aligned}
$$

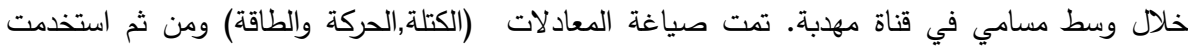

$$
\begin{aligned}
& \text { فرضية الطول الموجي الطويل وعدد رينولد الصغير للتبسيط. تم الحصول على حقل السرعة, تدرج الضغط, } \\
& \text { درجة الحرارة وخطوط الجريان عند تطبيق تقنية الاضطراب لحل المعادلات التفاضلية الجزئية اللاخطية . } \\
& \text { أظهرت الدراسة أن السرعة تنخفض مع عدد هارتمان بينما تتعزز مع معلمة المسامية. }
\end{aligned}
$$

\section{Introduction}

Fluid transmission in the form of a metachronal wave is called a peristaltic transmission. It is an important phenomenon in the transport of fluids to many organs, as in the motion of food in the esophagus during the swallowing, blood flow from the heart to all over the body, transport of the urine from the kidney to bladder, and in industrial and biological tools, such as heart-lung machines. Many researchers have interest in studying the peristalsis flow and its relationship to the engineering and biochemical systems, where they studied and analyzed the peristaltic motion of Newtonian and nonNewtonian fluids in all cases [1-3].

The Rabinowitsch fluid model has the characteristics of pseudoplastic fluids , known as shear-thinning fluids such as blood, Ketchup, and whipped cream. The Rabinowitsch fluid model significantly 
describes the influence of lubricant additives and agrees with empirical facts for shear rate on a wide range. Through the previous years, scientists have tried very hard to enhance the efficiency of stability specifications of non-Newtonian lubricants. The viscosity of this model indicates a nonlinear relationship between the shearing stress and shearing strain rate. The importance of the model lies in the three main classifications of the various values for Pseudo-plastic parameter $\kappa$, i.e., for Pseudoplastic fluid, the value of $\kappa$ is larger than zero, and in a Newtonian fluid, it is equal to zero, while in Dilatant nature (shear-thickening) it is smaller than zero. Pumping of blood in heart/lung machines is regarded as an application of this model in the peristaltic flow which is very beneficial in biomedicine and physiology. Akbar [4] presented studies in the Rabinowitsch fluid under the effect heat transfer in a circular tube. Singh [5] analyzed the application of Rabinowitsch fluid model in peristaltic flow through a uniform tube. Vaidya [6] discussed variable fluid characteristics inside a compliant wall on the peristaltic flow of Rabinowitsch fluid in an inclined tube. Ali [7] and Maraj [8] depicted the flow of Rabinowitsch fluid through a curved channel. Kumare [9] analyzed the effect of slip condition on the peristaltic flow of Rabinowitsch fluid in the non-uniform channel. Many other studies about using the Rabinowitsch fluid have been also reported [10-15].

The study of the effective magnetic field through a fluid in the channel has become an increasingly attractive research area [16,17]. Over the past few decades, distinguished researchers have extensively worked on the dynamics of fluid in the existence of a magnetic field. They have made an endeavor to include it in the industry, medical technology, and bio-engineering. Several authors [18-20] studied MHD influence and porous medium in peristalsis flow. Shaheen [21] discussed MHD effect and heat transfer along with the effect of the Brownian motion on the peristaltic flow of Jeffrey six -constant fluid.

Cilia are tiny hair-like structures that are presented in several organs in the human body. The motion of cilia plays a significant part in physiological processes like reproduction, circulation, and feeding. Many studies related to cilia transport have been executed [22-28].

In this article, we discuss the effects of the magnetic field, heat transfer and porous medium on incompressible laminar flow for non-Newtonian Rabinowitsch fluid in a circular channel when the inner of which is ciliated. This paper is organized as follows: in section 2, we presented the specifics of the physical formulation of our problem. The perturbation method used to solve our system equations is given in section 3. The numerical results were studied by plotting diagrams in section 4 . In the last section, all results were summarized and discussed.

\section{Mathematical formulation}

Let us take in our consideration the ciliary motion phenomenon for the two-dimensional flow of an incompressible Rabinowitsch fluid in the symmetric tube. $c$ denotes the constant speed due to the beating of cilia along the tube whose inner surface is ciliated. We have considered a porous medium and assumed that $\beta_{0}$ is a magnetic field. The problem geometry is exhibited in the cylindrical system $(\bar{R}, \bar{Z})$ where $\bar{Z}$ - axis is located along the centerline of the channel and $\bar{R}$ is orthogonal on the channel.

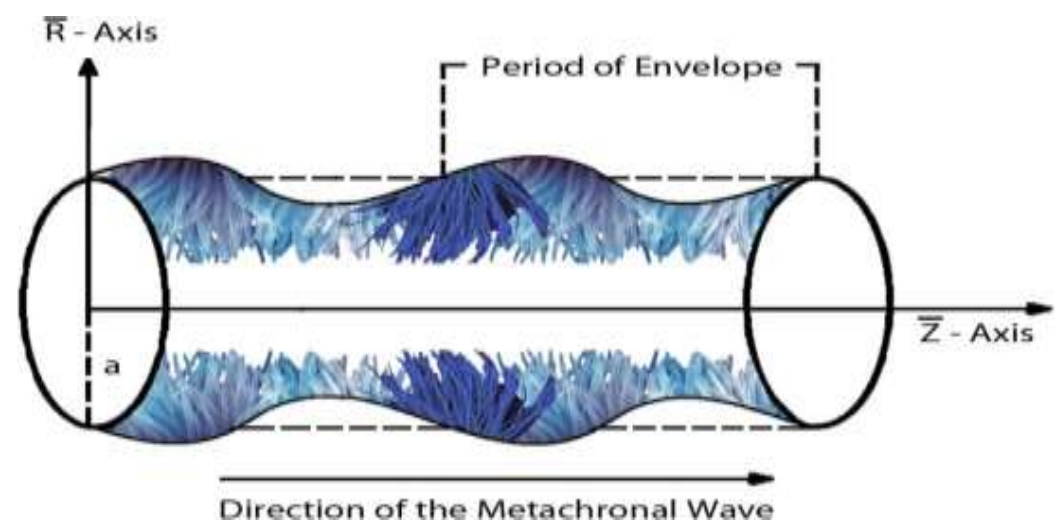

The governing equations (the continuity equation, motion equation, and energy equation) for incompressible fluid are described with respect to the laboratory frame $(\bar{R}, \bar{Z}, \bar{t})$, as follows:

$\frac{\partial \bar{U}}{\partial \bar{R}}+\frac{\bar{U}}{\bar{R}}+\frac{\partial \bar{W}}{\partial \bar{Z}}=0$ 
$\rho\left(\frac{\partial \bar{U}}{\partial \bar{t}}+\bar{U} \frac{\partial \bar{U}}{\partial \bar{R}}+\bar{W} \frac{\partial \bar{U}}{\partial \bar{Z}}\right)=-\frac{\partial \bar{P}}{\partial \bar{R}}+\frac{1}{\bar{R}} \frac{\partial}{\partial \bar{R}}\left(\bar{R}_{\bar{S}} \bar{R} \bar{R}\right)+\frac{\partial}{\partial \bar{Z}}\left(\bar{S}_{\bar{R} \bar{Z}}\right)-\frac{\bar{S}_{\bar{\theta}} \bar{\theta}}{\bar{R}}-\frac{\mu}{G} \bar{U}$

$\rho\left(\frac{\partial \bar{W}}{\partial \bar{t}}+\bar{U} \frac{\partial \bar{W}}{\partial \bar{R}}+\bar{W} \frac{\partial \bar{W}}{\partial \bar{Z}}\right)=-\frac{\partial \bar{P}}{\partial \bar{Z}}+\frac{1}{\bar{R}} \frac{\partial}{\partial \bar{R}}\left(\bar{R} \bar{S}_{\bar{R} \bar{Z}}\right)+\frac{\partial}{\partial \bar{Z}}\left(\bar{S}_{\bar{Z} Z}\right)-\frac{\mu}{G} \bar{W}-\sigma \beta_{0}^{2} \bar{W}$

$\rho c_{p}\left(\frac{\partial \bar{T}}{\partial \bar{t}}+\bar{U} \frac{\partial \bar{T}}{\partial \bar{R}}+\bar{W} \frac{\partial \bar{T}}{\partial \bar{Z}}\right)=\frac{\partial \bar{U}}{\partial \bar{R}}+\left(\bar{S}_{\bar{R} \bar{Z}}\right) \frac{\partial \bar{W}}{\partial \bar{R}}+\left(\bar{S}_{\bar{Z} \bar{R}}\right) \frac{\partial \bar{U}}{\partial \bar{Z}}+\frac{\partial \bar{W}}{\partial \bar{Z}}\left(\bar{S}_{\bar{Z} Z}\right)+K\left(\frac{\partial^{2} \bar{T}}{\partial \bar{R}^{2}}+\frac{1}{\overline{\bar{R}}} \frac{\partial \bar{T}}{\partial \bar{R}}+\frac{\partial^{2} \bar{T}}{\partial \bar{Z}^{2}}\right)$

where $\rho$ is the density, $\bar{W}$ and $\bar{U}$ denote velocity components in the axial $\bar{Z}$ and radial $\bar{R}$ directions in the fixed frame, respectively, $\bar{P}$ is the pressure, $\beta_{0}$ is an applied magnetic field, $\bar{T}$ is the temperature, $K$ is the thermal conductivity, $\mu$ is the fluid viscosity, and $G$ is the porosity parameter . The stress tensor formulation for the Rabinowitsch fluid is:

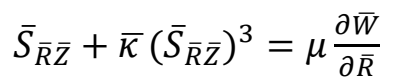

Where $\bar{\kappa}$ is the Pseudo-plasticity coefficient. The Mathematical expression for The envelops of the

cilia tips is:

$\bar{R}=\bar{H}=\bar{f}(\bar{Z}, \bar{t})=a+a \epsilon \cos \left(\frac{2 \pi}{\lambda}(\bar{Z}-c \bar{t})\right)$

$\bar{Z}=\bar{g}\left(\bar{Z}, \bar{Z}_{0}, \bar{t}\right)=a+a \epsilon \alpha \sin \left(\frac{2 \pi}{\lambda}(\bar{Z}-c \bar{t})\right)$

where $a$ refers to the radius of the channel, $\epsilon$ denotes non-dimensional measure according to the cilia length, $\lambda$ is the wavelength, $\bar{Z}_{0}$ is the particle position, $c$ is wave velocity, and $\alpha$ is the measure of the eccentricity of the elliptical motion. With no-slip condition applied, the cilia tips are the responsible of causing the velocities of the transporting fluid, which can be given as [4]:

$\bar{W}=\frac{\partial \bar{Z}}{\partial \bar{t}_{\bar{Z}_{0}}}=\frac{\partial \bar{g}}{\partial \bar{t}}+\frac{\partial \bar{g}}{\partial \bar{Z}} \frac{\partial \bar{Z}}{\partial \bar{t}}=\frac{\partial \bar{g}}{\partial \bar{t}}+\frac{\partial \bar{g}}{\partial \bar{Z}} \bar{W}$

$\bar{U}=\frac{\partial \bar{R}}{\partial \bar{t}_{\bar{Z}_{0}}}=\frac{\partial \bar{f}}{\partial \bar{t}}+\frac{\partial \bar{f}}{\partial \bar{Z}} \frac{\partial \bar{Z}}{\partial \bar{t}}=\frac{\partial \bar{f}}{\partial \bar{t}}+\frac{\partial \bar{f}}{\partial \bar{Z}} \bar{W}$

By utilizing Eq. (6) and (7) into Eq. (8) and (9), we get

$\bar{W}=\frac{\frac{-2 \pi}{\lambda}\left(\epsilon \alpha a c \cos \left(\frac{2 \pi}{\lambda}\right)(\bar{Z}-c \bar{t})\right)}{1-\frac{2 \pi}{\lambda}\left(\epsilon \alpha a \cos \left(\frac{2 \pi}{\lambda}\right)(\bar{Z}-c \bar{t})\right)}$

$\bar{U}=\frac{\frac{2 \pi}{\lambda}\left(\epsilon a c \sin \left(\frac{2 \pi}{\lambda}\right)(\bar{Z}-c \bar{t})\right)}{1-\frac{2 \pi}{\lambda}\left(\epsilon \alpha a \cos \left(\frac{2 \pi}{\lambda}\right)(\bar{Z}-c \bar{t})\right)}$

$(\bar{R}, \bar{Z})$ represents the fixed frame, which is an unsteady flow between two channels, while the wave frame $(\bar{r}, \bar{Z})$ is moving with the wave speed, so that the flow is considered steady. The transformation between the fixed frame and the wave frame is:

$\bar{r}=\bar{R}, \quad \bar{z}=\bar{Z}-c \bar{t}, \quad \bar{u}=\bar{U}, \quad \bar{w}=\bar{W}-c$

The non-dimensional quantities are as below:

$$
\begin{aligned}
& r=\frac{\bar{r}}{a}, \quad z=\frac{\bar{z}}{\lambda}, \quad w=\frac{\bar{w}}{c}, \quad u=\frac{\lambda \bar{u}}{a c}, \quad p=\frac{a^{2} \bar{p}}{c \lambda \mu}, \quad \theta=\frac{\left(\bar{T}-\bar{T}_{0}\right)}{\bar{T}_{0}}, \quad t=\frac{c \bar{t}}{\lambda}, \quad \delta=\frac{a}{\lambda}, \quad P_{r}=\frac{\mu c_{p}}{K}, \quad E_{C}= \\
& \frac{c^{2}}{c_{p} T_{0}}, B_{r}=E_{c} P_{r}, S_{i j}=\frac{a \bar{s}_{i j}}{c \mu}, \quad \kappa=\frac{\bar{\kappa} \mu^{2} c^{2}}{a^{2}}, M=\sqrt{\frac{\sigma}{\mu}} \beta_{0} a, \quad g=\frac{G}{a^{2}}, \quad n=\frac{1}{g}+M^{2}
\end{aligned}
$$

where $M$ is Hartmann number, $R_{e}, B_{r}$ and $P_{r}$ are Reynolds, Brikmann and Prandlt numbers, respectively, and $g$ is the porosity parameter.

With the presumption for low Reynolds number and long wavelength, Eqs. (1) - (5) with the help of Eq. (10) and Eq. (11) become:

$$
\begin{aligned}
& \frac{\partial u}{\partial r}+\frac{u}{r}+\frac{\partial w}{\partial z}=0 \\
& \frac{\partial p}{\partial r}=0 \\
& -\frac{\partial p}{\partial z}+\frac{1}{r} \frac{\partial}{\partial r}\left(r S_{r z}\right)-\frac{1}{g}(w+1)-M^{2}(w+1)=0 \\
& \frac{1}{r} \frac{\partial}{\partial r}\left(r \frac{\partial \theta}{\partial r}\right)+B_{r} S_{r z} \frac{\partial w}{\partial r}=0 \\
& S_{r z}+\kappa\left(S_{r z}\right)^{3}=\frac{\partial w}{\partial r}
\end{aligned}
$$

The dimensionless form of the boundary condition is derived as: 


$$
\left.\begin{array}{rl}
\frac{\partial w}{\partial r}=0, & \frac{\partial \theta}{\partial r}=0, \psi=0 \quad \text { at } r=0 \\
w=\frac{-2 \lambda \epsilon \alpha \beta \cos (2 \pi z)}{1-2 \lambda \epsilon \alpha \beta \cos (2 \pi z)}-1, \quad \theta=0, \psi=Q \quad \text { at } \quad r=h=1+\epsilon \cos (2 \pi z)
\end{array}\right\}
$$

\section{Solution methodology}

With utilizing the relationship between the stream function and the velocity

$$
w=\frac{1}{r} \frac{\partial \psi}{\partial r}
$$

substituting Eq. (18) into Eq. (14), the equation becomes

$$
-\frac{\partial p}{\partial z}+\frac{1}{r} \frac{\partial}{\partial r}\left(r S_{r z}\right)-N\left(\frac{1}{r} \frac{\partial \psi}{\partial r}+1\right)=0
$$

where $N=\frac{1}{g}+M^{2}$. The following equation is obtained when integrating Eq.(19):

$$
S_{r z}=N \frac{\psi}{r}+\frac{r}{2}\left(N+\frac{\partial p}{\partial z}\right)
$$

Now, by employing Eq. (18) and (20) in Eq. (16), we obtain the following

$$
N \frac{\psi}{r}+\frac{r}{2}\left(N+\frac{\partial p}{\partial z}\right)+\kappa\left(N \frac{\psi}{r}+\frac{r}{2}\left(N+\frac{\partial p}{\partial z}\right)\right)^{3}=\frac{\partial}{\partial r}\left(\frac{1}{r} \frac{\partial \psi}{\partial r}\right)
$$

Eq. (21) is difficult to be solved exactly, therefore we resort to using the perturbation method to solve this equation. In order to obtain the perturbed solution about the stream function $\psi$, we use the following expansion[29,30].

$\psi=\psi_{0}+N \psi_{1}+\cdots$

The following system was obtained by substituting Eq.(22) into(21) and gathering the coefficients of the same powers of $N$.

\subsection{Zero-order system}

$$
\frac{r}{2} \frac{\partial p}{\partial z}+\kappa \frac{r^{3}}{8} \frac{\partial p^{3}}{\partial z}+\frac{1}{r} \frac{\partial^{2} \psi_{0}}{\partial r^{2}}-\frac{1}{r^{2}} \frac{\partial \psi_{0}}{\partial r}=0
$$

associated with the following boundary conditions

$$
\begin{array}{ll}
\psi_{0}=0, & \frac{\partial}{\partial r}\left(\frac{1}{r} \frac{\partial \psi_{0}}{\partial r}\right)=0, \quad \text { at } r=0 \\
\psi_{0}=Q_{0}, & \frac{\partial \psi_{0}}{\partial r}=r\left(\frac{-2 \pi \alpha \epsilon \beta \operatorname{Cos}[2 \pi z]}{1-2 \pi \alpha \epsilon \beta \operatorname{Cos}[2 \pi z]}-1\right), \quad \text { at } r=h .
\end{array}
$$

\subsection{First-order system}

$$
\frac{\psi_{0}}{r}+\frac{r}{2}+\frac{3}{4} \kappa r \frac{\partial p^{2}}{\partial z} \psi_{0}+\frac{3}{8} \kappa r^{3} \frac{\partial p^{2}}{\partial z}-\frac{1}{r} \frac{\partial^{2} \psi_{1}}{\partial r^{2}}+\frac{1}{r^{2}} \frac{\partial \psi_{1}}{\partial r}=0
$$

Associated with the following boundary conditions

$$
\begin{aligned}
& \psi_{1}=0, \quad \frac{\partial}{\partial r}\left(\frac{1}{r} \frac{\partial \psi_{1}}{\partial r}\right)=0, \quad \text { at } r=0 \\
& \psi_{1}=Q_{1}, \quad \frac{\partial \psi_{1}}{\partial r}=0, \quad \text { at } r=h
\end{aligned}
$$

By solving the zero and first-order systems using the Mathematica program, the final closed-form solution for stream function, velocity and temperature, will be found as follows:

$$
\begin{gathered}
\psi=\frac{r^{4} \frac{d p}{d z}}{16}+\frac{1}{192} r^{6} \frac{d p^{3}}{d z} \kappa+\frac{1}{64} r^{2}\left(-32+32 j-8 h^{2} \frac{d p}{d z}-h^{4} \frac{d p^{3}}{d z} \kappa\right)+N\left(\frac{5 r^{8} \frac{d p^{3}}{d z} \kappa}{4608}+\frac{r^{10} \frac{d p^{5}}{d z} \kappa^{2}}{20480}+\right. \\
\frac{1}{512} r^{4}\left(32 j-\quad 8 h^{2} \frac{d p}{d z}-\quad h^{4} \frac{d p^{3}}{d z} \kappa\right)-\frac{r^{6} \frac{d p}{d z}\left(-16-96 j \frac{d p}{d z} \kappa+24 h^{2} \frac{d p^{2}}{d z} \kappa+3 h^{4} \frac{d p^{4}}{d z} \kappa^{2}\right)}{6144}+\cdots \\
w=\frac{1}{r}\left(\frac{r^{3} \frac{d p}{d z}}{4}+\frac{1}{32} r^{5} \frac{d p^{3}}{d z} \kappa+\frac{1}{32} r\left(-32+32 j-8 h^{2} \frac{d p}{d z}-h^{4} \frac{d p^{3}}{d z} \kappa\right)+N\left(\frac{5}{576} r^{7} \frac{d p^{3}}{d z} \kappa+\frac{r^{9} \frac{d p^{5}}{d z} \kappa^{2}}{2048}+\right.\right. \\
\quad \frac{1}{128} r^{3}\left(32 j-8 h^{2} \frac{d p}{d z}-h^{4} \frac{d p^{3}}{d z} \kappa\right)-\cdots
\end{gathered}
$$$$
\theta=\frac{1}{832359628800}\left(13005619200 B h^{4} j^{2} N^{2}-2528870400 B h^{6} j^{2} N^{3}-13005619200 B_{r} j^{2} N^{2} r^{4}+\right.
$$$$
3251404800 B h^{2} j^{2} N^{3} r^{4}-722534400 B_{r} j^{2} N^{3} r^{6}+26011238400 B_{r} h^{4} j N \frac{d p}{d z}-\cdots
$$

where $h=1+\epsilon \operatorname{Cos}[2 \pi z] \quad$ and $\quad j=\frac{-2 \pi \alpha \epsilon \beta \operatorname{Cos}[2 \pi z]}{1-2 \pi \alpha \epsilon \beta \operatorname{Cos}[2 \pi z]}$

The formula of the heat transfer coefficient is given by the following: 


$$
\mathrm{Z}=\left.\frac{\partial h}{\partial z}\left(\frac{\partial \theta}{\partial r}\right)\right|_{r=h}
$$

\subsection{Volume flow rate}

The fixed frame of the instantaneous volume flow rate is defined by:

$\bar{Q}=2 \pi \int_{0}^{\bar{H}} \bar{R} \bar{W} d \bar{R}$

where $\bar{H}$ is a function of $\bar{Z}$ and $\bar{t}$. Substituting the time relaxed system Eq. (10) into Eq. (11) and integrating yields

$\bar{Q}=\bar{q}+\pi c \bar{h}^{2} \quad$ where

$\bar{q}=2 \pi \int_{0}^{\bar{h}} \bar{r} \bar{w} d \bar{r}$

Whereas the flow is steady in the wave frame. The formulation of volume flow rate when using the dimensional variable becomes:

$Q=\frac{\bar{q}}{a^{2} c}=\int_{0}^{h} r w d r$

And taking the time-mean flow over a period $\left(T=\frac{\lambda}{c}\right)$ at a fixed $\bar{Z}$, the position is defined as

$\bar{F}=\frac{1}{T} \int_{0}^{T} \bar{Q} d \bar{t}$

Now, by substituting Eq. (34) into (36) and integrating, we get

$\frac{\bar{F}}{2 \pi a^{2} c}=\frac{\bar{q}}{2 \pi a^{2} c}+\frac{1}{2}\left(1+\frac{\epsilon^{2}}{2}\right)$

Introducing the non-dimensional time -mean flow $F$ and $Q$, respectively, in the fixed and wave frames of the coordinate, yields

$F=\frac{\bar{F}}{2 \pi a^{2} c} \quad, \quad Q=\frac{\bar{q}}{2 \pi a^{2} c}$

We rewrite Eq. (37) as

$F=Q+\frac{1}{2}\left(1+\frac{\epsilon^{2}}{2}\right)$

Now, we can employ Eq.(35) to obtain the pressure gradient $\frac{d p}{d z}$. The result of solution for volumetric flow flux is obtained as follows:

$$
\begin{aligned}
Q= & \left(-\frac{h^{2}}{2}+\frac{h^{2} j}{2}-\frac{1}{16} h^{4} j N^{2}-\frac{h^{4} \frac{d p}{d z}}{16}+\frac{1}{96} h^{6} N^{2} \frac{d p}{d z}-\frac{1}{32} h^{6} j N^{2}{\frac{d p^{2}}{d z}}^{2}-\frac{1}{96} h^{6} \frac{d p}{d z}^{3} \kappa+\frac{5}{768} h^{8} N^{2}{\frac{d p^{3}}{d z}}^{3} \kappa+\right. \\
& \left.\frac{h^{10} N^{2} \frac{d p^{5}}{d z} \kappa^{2}}{1280}\right)
\end{aligned}
$$

The following procedure was applied when using the perturbation method to calculate the value of the pressure gradient. For small values of $N \ll 1$,

$\frac{d p}{d z}=\left(\frac{d p}{d z}\right)_{0}+N\left(\frac{d p}{d z}\right)_{1+\cdots}$

By substituting Eq.(40) into Eq.(39) and gathering the coefficients of the same powers of $\kappa$, one can obtain the following systems.

\subsection{Zero-order system}

$$
\left(-\frac{h^{2}}{2}+\frac{h^{2} j}{2}-\frac{1}{16} h^{4} j N-\frac{h^{4}\left(\frac{d p}{d z}\right)_{0}}{16}+\frac{1}{96} h^{6} N\left(\frac{d p}{d z}\right)_{0}\right)-Q=0
$$

\subsection{First-order system}

$$
\begin{aligned}
& \left(-\frac{1}{16} h^{4} j-\frac{h^{4}}{16}\left(\frac{d p}{d z}\right)_{1}+\frac{1}{96} h^{6}\left(\frac{d p}{d z}\right)_{0}-\frac{1}{32} h^{6} j\left(\frac{d p}{d z}\right)_{0}{ }^{2} \kappa-\frac{3}{96} h^{6}\left(\frac{d p}{d z}\right)_{0}{ }^{2}\left(\frac{d p}{d z}\right)_{1} \kappa+\frac{5}{768} h^{8}\left(\frac{d p}{d z}\right)_{0}^{3} \kappa+\right. \\
& \left.\frac{h^{10} \kappa^{2}}{1280}\left(\frac{d p}{d z}\right)_{0}{ }^{5}\right)=0
\end{aligned}
$$

By solving the Zero and First-order systems, we get a final form for pressure gradient which is given as follows:

$$
\begin{aligned}
& \frac{\partial p}{\partial z}=\frac{2^{2 / 3} h^{4}}{\left(B_{5}\right)^{1 / 3}}-\frac{2^{1 / 3}\left(B_{5}\right)^{1 / 3}}{h^{6} \kappa}+\left(N ^ { 2 } \left(240 h^{4} j+\cdots\right.\right. \\
& B_{5}=-12 h^{14}(-1+j) \kappa^{2}+24 h^{12} Q \kappa^{2}+\sqrt{2} B_{6} \\
& B_{6}=\sqrt{h^{24} \kappa^{3}\left(h^{6}+72 h^{4}(-1+j)^{2} \kappa-288 h^{2}(-1+j) Q \kappa+288 Q^{2} \kappa\right)}
\end{aligned}
$$




\section{Numerical results and discussion}

This section is dedicated to discuss the graphical results for fluid velocity, pressure gradient, temperature profile, and stream function. In this work, we are interested in analyzing the influences of various parameters on fluid flow.

\subsection{Velocity Profile}

The conduct of the velocity profile for values of $M, g, \alpha$ and coefficient of Pseudo-plasticity is presented in Figure-1 . It is noticed that the velocity magnitude is utmost in the centerline of the tube, for all parameters. Figure-1.a represents the effect of the coefficient of Pseudo-plasticity on the velocity of the fluid. It is observed that the velocity with Newtonian fluid becomes maximal and is decreased with the Pseudo-plasticity, while for the Dilatant fluid, it behaves differently such that the velocity field is increases near the wall whilst decreases at the center of the channel. Figure-1.b displays the effect of the permeability parameter $g$ on the velocity. It is evident that the velocity is enhanced when $g$ increases. Figure-1.c reveals that the increase of $M$ leads to a decrease in velocity axial. Figure-1.d shows the effect of the eccentricity of the cilia elliptic path $\alpha$ on the velocity of the fluid. When the value of $\alpha$ increases, the velocity of the fluid is falling.

(a)

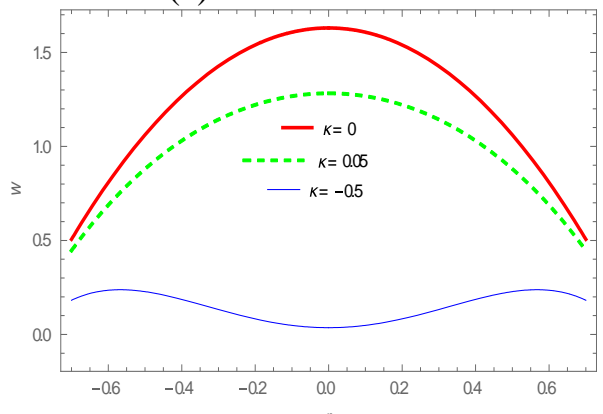

(c)

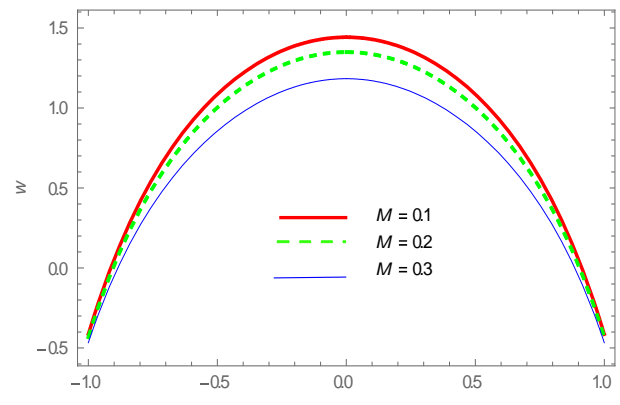

(b)

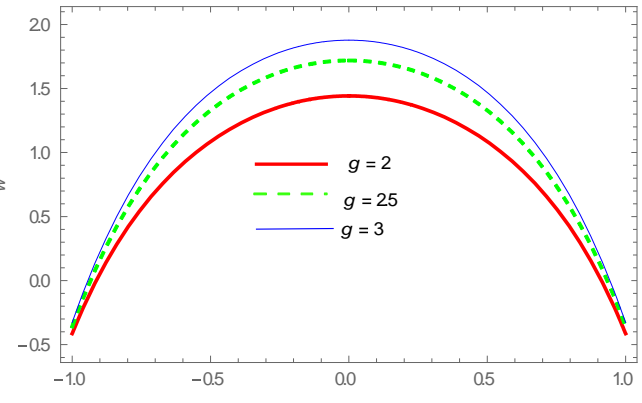

(d)

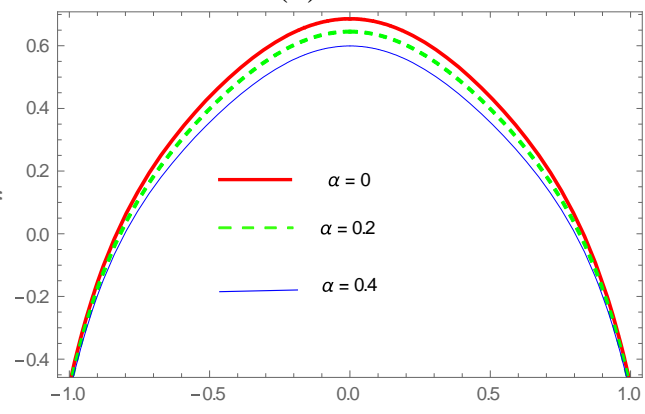
Figure-1 Velocity profiles $w(r)$ for various values of (a) coefficient of Pseudo-plasticity $\kappa$ (b)
permeability parameter $g$, (c) Hartman number $M$, (d) the measure of the eccentricity of the
elliptical motion parameter $\alpha$, and other parameters are $(\epsilon=0.1, \beta=0.1, F=0.5, z=0.02)$ Figure-1 Velocity profiles $w(r)$ for various values of (a) coefficient of Pseudo-plasticity $\kappa$ (b)
permeability parameter $g$, (c) Hartman number $M$, (d) the measure of the eccentricity of the
elliptical motion parameter $\alpha$, and other parameters are $(\epsilon=0.1, \beta=0.1, F=0.5, z=0.02)$

\subsection{Pressure Gradient}

Figure-2 shows the behavior of pressure gradient with $z$ for different values of, $M, \alpha, g$ and the coefficient of Pseudo-plasticity. It is clear that the pressure gradient exhibits a fluctuating behavior, reaching its maximum values at $z=-1,0,1, \ldots$, while approaching its minimum values at $z=$ $-0.5,0.5, \ldots$. This indicates that a high level of flow can pass with no need for large pressure gradient in the channel. Figure.a illustrates the pressure gradient which is robustly affected by the increase in $g$. On the other hand, Figure-2.b displays a simple decrease in pressure gradient with the increase of $M$. Figure-2.c.d demonstrates that when the values of $\alpha$ and $\beta$ increase the pressure gradient value is increased at $(-0.75 \leq z \leq-0.25)$ and $(0.25 \leq z \leq 0.75)$, but the results are reversed at $(-1 \leq z \leq$ $-0.75)$ and $(-0.25 \leq z \leq 0.25)$, approximately. 
(a)

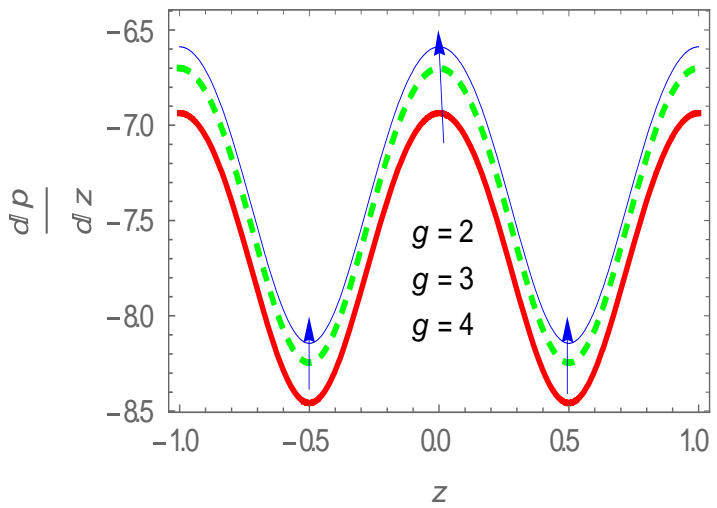

(c)

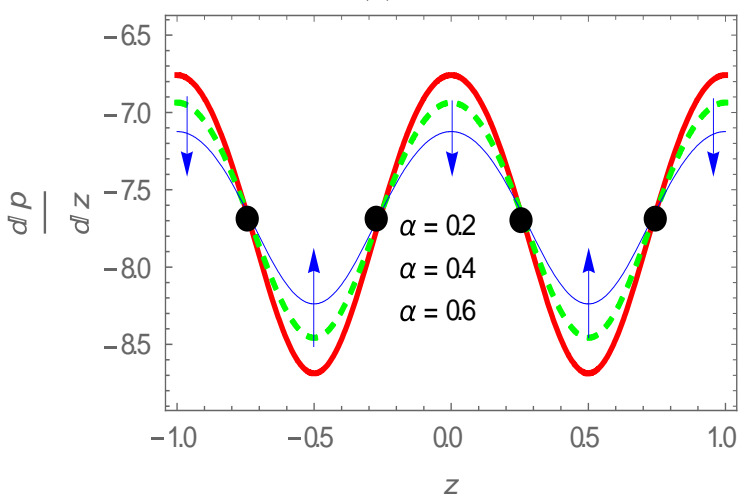

Figure-2 Pressure profiles $\frac{d p}{d z}$ for various values of number $M$,(c) the measure of the eccentricity of the elliptical motion parameter $\alpha$ (d) wave number $\beta$, and for other fixed parameters $(\epsilon=0.1, \kappa=0.2, F=0.1)$.

\subsection{Trapping}

Trapping is an interesting phenomenon of peristalsis in which streamlines split to trap a bolus in the wave frame. Figure-3.a deduces that increasing the value of permeability parameter $g$ causes an increase in the size of trapping, while the number of the trapping is decreased. Figure-3.b indicates that the trapped bolus size was shrinked but the number increases by increasing $\alpha$. Figure-3.c shows that the number and size of the trapped bolus were increased by increasing $M$. Figure-3.d shows the effect of the Pseudo-plasticity coefficient on the streamlines. We noticed that for Dilatant nature the size of the trapping bolus is smaller than that of the Pseudo-plasticity fluid, but the number of the trapping is higher than the number of the Pseudo-plasticity fluid. 
(A)

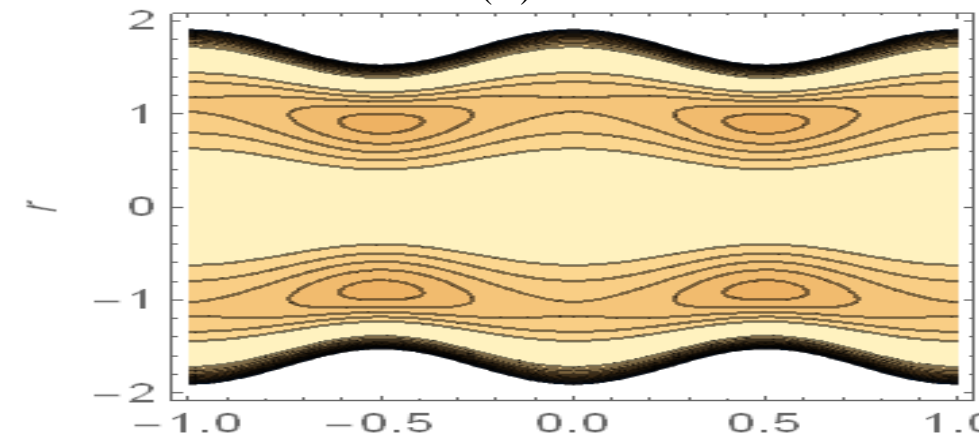

Z

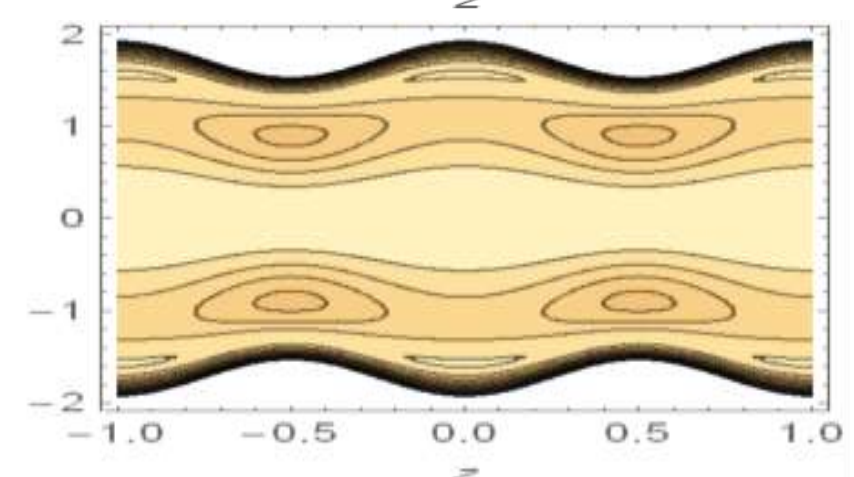

(B)

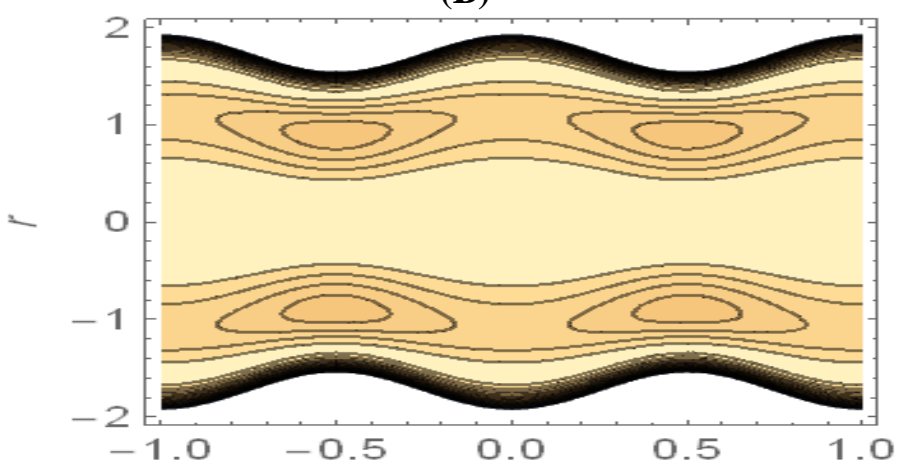

$Z$

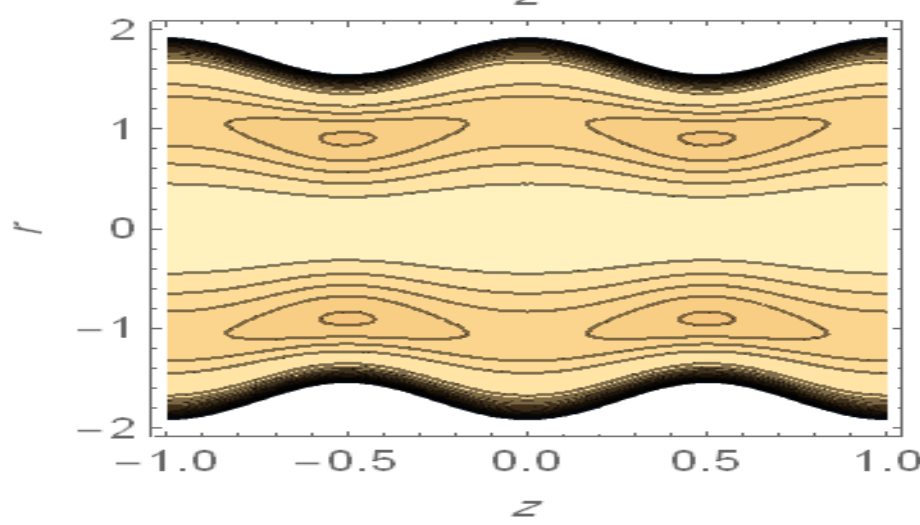


(c)
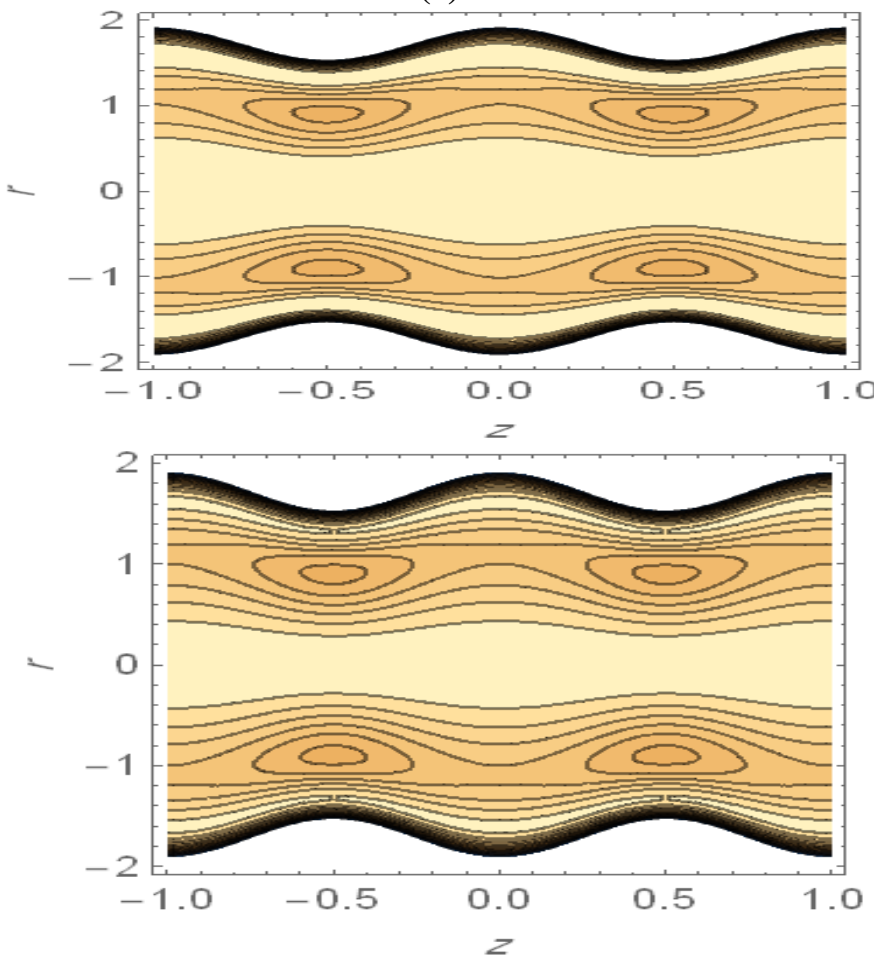

(d)

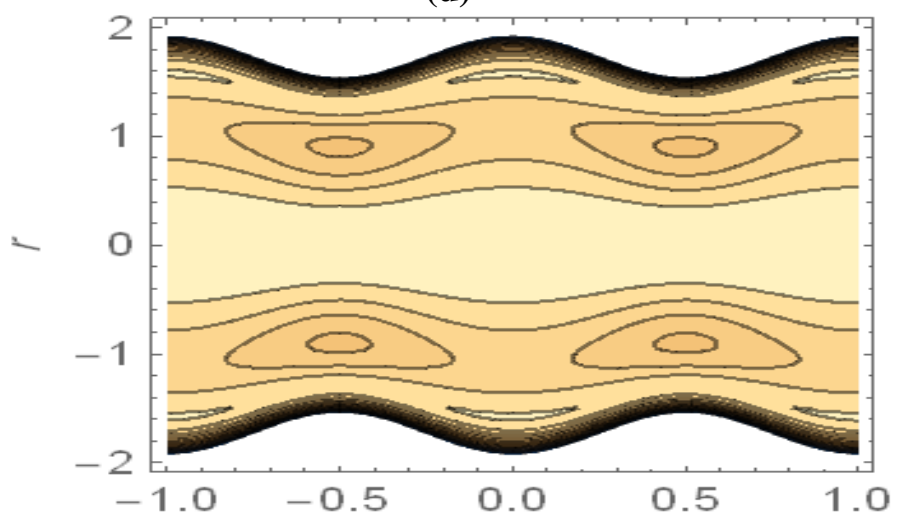

Z

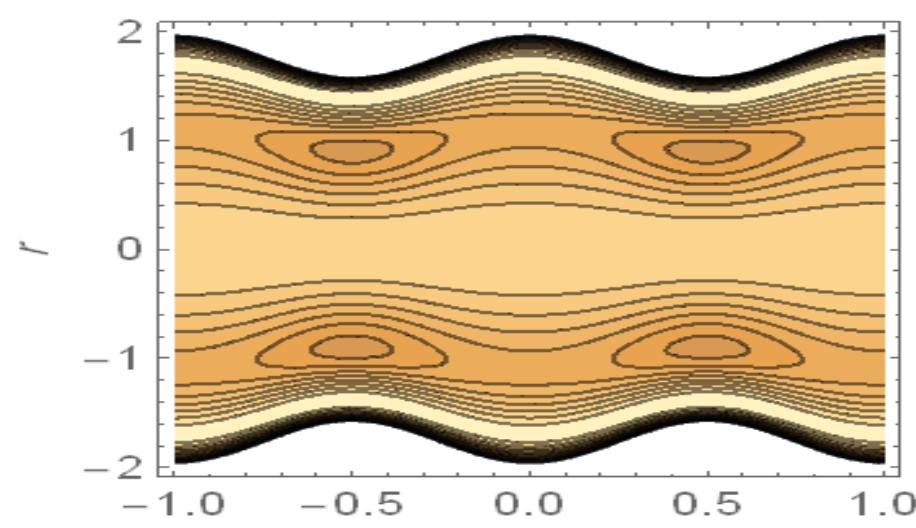

$z$

Figure 3-Stream line profiles for various values of (a) permeability parameter $g=\{2,4\}$,(b) the measure of the eccentricity of the elliptical motion parameter $\alpha=\{0.1,0.3\}$ (c) Hartman number $M=\{0.1,0.2\}$ (d) coefficient of Pseudo-plasticity $\kappa=\{-1,1\}$, and for other fixed parameters $(\epsilon=0.1, \beta=0.2, F=2)$. 


\subsection{Temperature}

The effect of all parameters on the temperature profile is illustrated in Figure- 4 , which depicts that, for all values of parameters, the temperature remains identical at the center of the tube. Figure-4.a shows that the temperature is increased at the wall for the Dilatant fluid, whilst it is decreased the Newtonian fluid and the Pseudo-plastic fluid. Figure-4.b illustrates that when the values of $M$, the temperature drops close to the wall until it reaches a certain value in the central part of the channel, where it stays stable. it is observed from Figure-4.c that when the $g$ increases, the temperature rises.Figure-4. d reveals that the cilia elliptical eccentricity path $\alpha$ does not affect the temperature of the fluid.

(a)

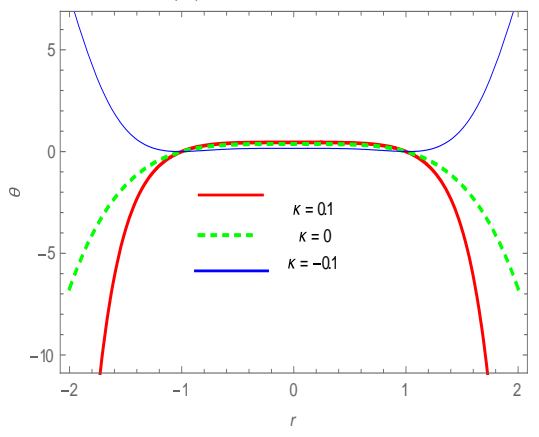

(c)

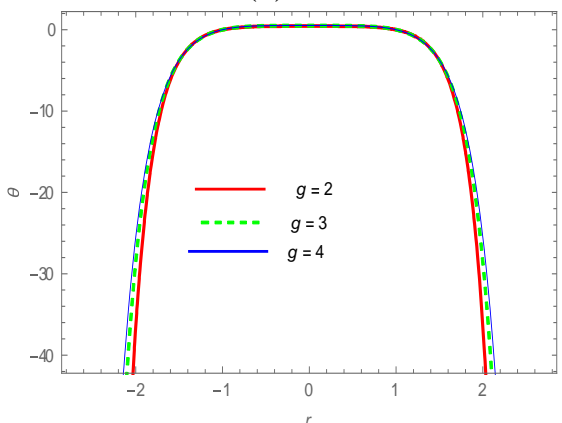

(b)

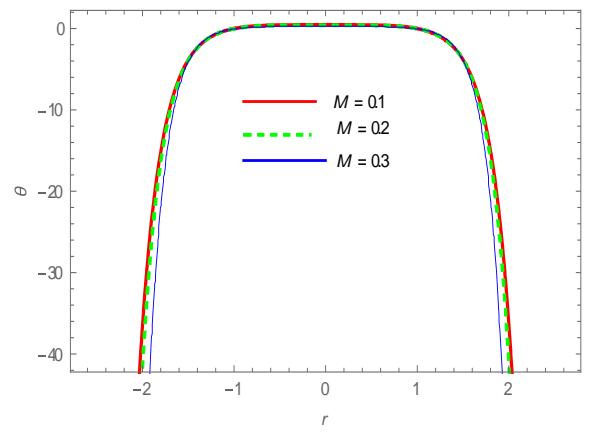

(d)

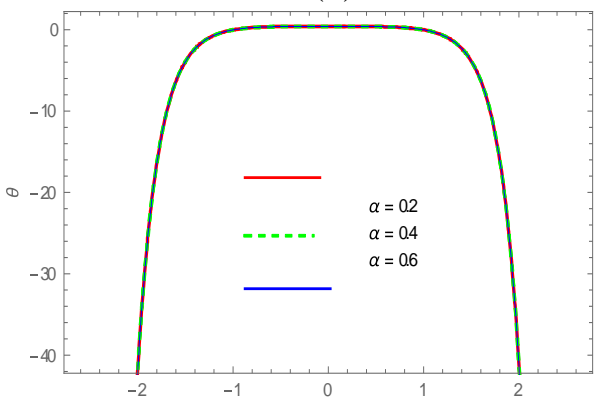

Figure 4-Temperature profiles $\theta(r, z)$ for various values of (a) coefficient of Pseudo-plasticity $\kappa$ (b) Hartman number $M$ (c) permeability parameter $g$ (d) the measure of the eccentricity of the elliptical motion parameter $\alpha$, and for other fixed parameters $F=0.1, \epsilon=0.1, \beta=0.2, B_{r}=0.5, z=0.75$

\subsection{Heat transfer coefficient}

Diverse values of the heat transfer coefficient at the wall for various quantities of the parameters are illustrated in Figure-5. Graphs reveal that, due to Metachronal wave along the walls, the conduct of $\mathrm{Z}$ is oscillatory in nature. Figure-5.a shows that the enhancement of the values of the $g$ the rate of heat transfer is increased. We notice through Figure-5.b that the heat transfer rate is maximum with the Newtonian fluid, while it is decreased with the the Pseudo-plastic fluid and reserves its behavior with the Dilatant fluid. Fig. (5.c) illustrates that the increase $M$ reduces the heat transfer rate. While Fig. (5.d) shows that the heat transfer rate increases with increases $\alpha$. 
(a)

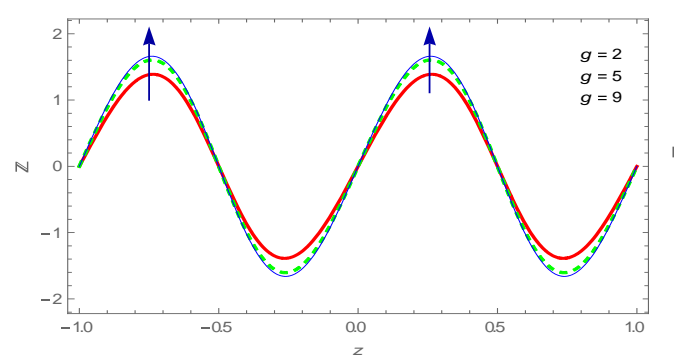

(c)

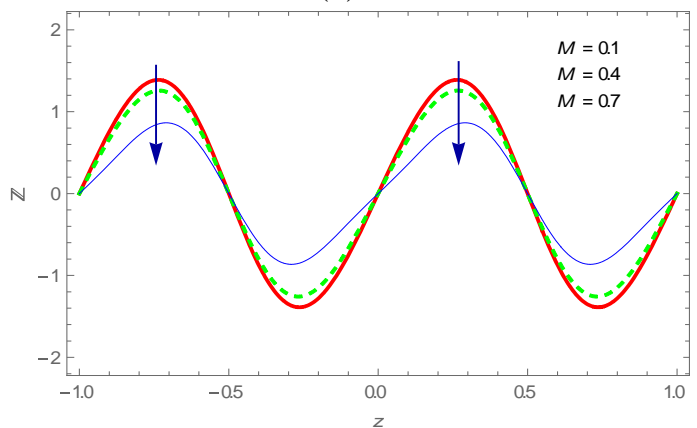

(b)

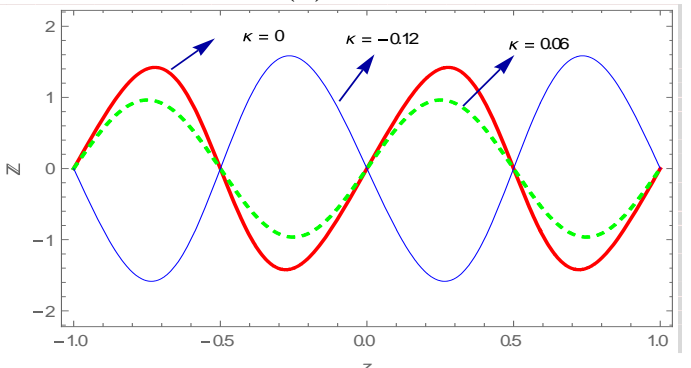

(d)

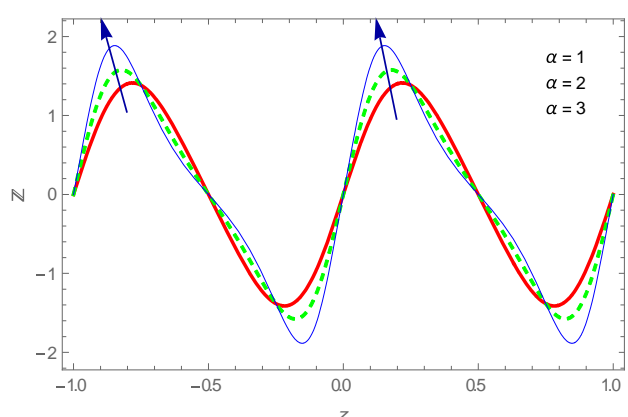

Figure 5-Heat transfer coefficient $\mathrm{Z}(\mathrm{z}$ ) for various values of (a) permeability parameter $g$ (b) coefficient of Pseudo-plasticity $\kappa$ (c) Hartman number $M$ (d) the measure of the eccentricity of the elliptical motion parameter $\alpha$, and for other fixed parameters $F=0.1, \epsilon=0.1, \beta=0.2, B_{r}=$ $0.5, r=0.5$.

\section{Conclusions}

In this paper, the heat transfers for the flow of the MHD fluid, known as the Pseudoplastic, along a circular channel with ciliated walls through a porous medium is analyzed. The major findings of the presented investigation are listed below.

The velocity profile shape is parabolic in nature and its maximum value appears in the central part of the channel, for all parameters' magnitudes and we notice that the velocity magnitude slows down with increasing the magnetic field and the eccentricity of the cilia elliptic path, whereas it is enhanced with increasing porosity parameter, and also observed that with Dilatant fluid, the velocity is low as compared with that of the Newtonian and Pseudoplastic fluids. The pattern of the temperature is the same at the centerline of the channel for all parameters. Increasing the value of the permeability parameter leads to an increase in the size and number of the trapping , and the number and size of the trapped bolus is increased by increasing the magnetic field. Also notice the size of the trapped bolus is reduced when the value of the cilia elliptic path eccentricity is increased. The diagram of the pressure gradient is oscillation; it is high near the wall and low in the center. The behavior of the heat transfer rate profile is oscillatory in nature; also at the walls, it has a wobbling motion for all embedded parameters.

\section{References}

1. Ali H. A. 2017. Influence of Magnetic field on Peristaltic Transport for Eyring- Powell fluid in A Symmetric Channel During a Porous Medium. Journal of Mathematical Theory and Modeling, 7(9).

2. Akbar N. Sh. And Nadeem S. 2014. Exact solution of peristaltic flow of biviscosity fluid in an endoscope: A note. Alexandria Engineering Journal, 53: 449-454.

3. Latham T.W. 1966. Fluid Motion in a Peristaltic pump. MS thesis MIT Cambridge, MA.

4. Akbar N. Sh. And Butt A. W. 2015. Heat transfer analysis of Rabinowitsch Fluid Flow due to Metachronal Wave of Cilia. Results in Physics, 5: 92-98.

5. Singh B.K., Dr. and Singh U.P. 2014. Analysis of Peristaltic Flow in a Tub: Rabinowitsch Fluid Model. International Journal of fluid engineering, 6(1): 1-8. 
6. Vaidya H., Rajashekhar C., Manjunath G. and Prasad K. V. 2019. Peristaltic Mechanism of a Rabinowitsch Fluid an inclined channel with complaint wall and variable liquid properties. Journal of the Brazilian Society of Mechanical Sciences and Engineering, 41: 52.

7. Ali N., Sajid M., Javid K. and Ahmed R. 2016. Peristaltic Flow of Rabinowitsch Fluid in a Curved Channel:Mathematical Analysis Revisite. Z. Naturforsch.

8. Maraj E.N. and Nadeem S. 2015. Application of Rabinowitsch Fluid Model for Mathematical Analysis of Peristaltic Flow in a Curved Channel. Z.Naturforsch . 70(7): 513-520.

9. Kumare K. Th. and Kavitha A. 2017. The Influence of Slip Effects on Peristaltic Transport of a Rabinowitsch Fluid Model in a non -uniform tube., materials science and engineering, 263: 062004.

10. Javorova J. and Angelova J. 2018. On the Modified Reynolds equation for Journal bearing in a case of non-Newtonian Rabinowitsch fluid model. MATEC Web of Conferences, 145: 03007.

11. Saravana R., Vajravelu K. and Sreenadh S. 2018. Influence of Compliant Walls and Heat Transfer on the Peristaltic Transport of a Rabinowitsch Fluid in an Inclined Channel. A Journal of Physical Sciences, Z. Naturforsch., 73(9).

12. Singh UP. Gupta RS. And Kapur VK. 2012. On the Steady Performance of annular hydrostatic thrust bearing: Rabinowitsch fluid model. ASME J. Tribol, 134: 1341,5.

13. Singh U.P., Medhavi A., Gupta R.S. and Bhatt S. Sh. 2017. Analysis of Peristaltic Transport of Non-Newtonian Fluids Through Non-Uniform Tubes: Rabinowitsch Fluid Model. A Journal of Physical Sciences, Z. Naturforsch.

14. Sadaf H. and Nadeem S. 2017. Analysis of Combined Convective and Viscous Dissipation Effect for Peristaltic flow of Rabinowitsch Fluid model. Journal of Bionic Engineering, 14: 182-190.

15. Sinch U.P. 2015. An Exact Solution of non-Newtonian Peristaltic flow in a tube: Rabinowitsch fluid model. J. Sci. Univ.Kelaniya, 10: 1-11.

16. Mekheimer K.S. 2004. Peristaltic Flow of Blood under Effect of a Magnetic field in a non-uniform channel. Appl. Math. Comput.,153: 763-777.

17. Reddy M.G. 2015. Heat and Mass transfer on Magneto hydrodynamic Peristaltic flow in a porous medium with partial slip. Alexandria engineering journal, 55: 1225-1234.

18. Kothandapani M. and Srinivas S. 2008. On the Influence of Wall Properties in the MHD Peristaltic Transport with heat transfer and porous medium. Phys. Lelt., A. 372: 4586-4591.

19. Ellahi R., Shivanian E., Abbasbandy S. and Hayat T. 2015. Analysis of some Magneto hydrodynamic flows of third order fluid saturating porous Space. J. Porous Media, 18(2): 89-98.

20. Ahmed T. Sh. 2018. Effect of Inclined Magnetic Field on Peristaltic Flow of Carreau Fluid through Porous Medium in an Inclined Tapered Asymmetric Channel. Al-Mustansiriyah Journal of Science, 29( 3).

21. Shaheen A. and Nadeem S. 2017. Metachronal wave analysis for non-Newtonian fluid under thermophoresis and Brownian motion effects. Results in Physics, 7: 2950-2957.

22. Brennen C. 1974. An Oscillating-Boundary-Layer theory for Ciliary Propulsion .J. Fluid Mech., 65: 799-824.

23. Agrawal H.L. and Anawaruddin. 1984. Cilia Transport of Bio-fluid with variable viscosity. Indian J. Pure Appl. Math.,15(10): 1128-1139.

24. Sleigh M.A. 1962.The Biology of Cilia and Flagella. New York, MacMillian.

25. Miller CE. 1967 . An Investigation of the Movement of Newtonian Liquids Initiated and sustained by the Oscillation of Mechanical Cilia Aspen Emphysema Conf. 21, 309..

26. Akbar N. Sh., Khan Z.H. and Nadeem S. 2014. Metachronal beating of cilia under influence of Hartmann layer and heat transfer.Phys. J. Plus, 129: 176.

27. Akbar N. Sh. And Khan Z.H. 2015. Metachronal beating of Cilia under influence of Casson fluid and magnetic field. Appl. Math. Comput., 378: 320-326.

28. Abo-Elkhair R.E., Mekheimer Kh.S. and MoawadA .M.A. 2017.Cilia Walls influence on peristaltically induced motion of magnetic-fluid through a porous medium at moderate Reynolds number: Numerical study. Journal of the Egyptian Mathematical Society, 25: 238-251.

29. Awati V. B., Jyoti M. and Prasad K.V. 2017. Series analysis for the flow between two stretchable disks. En. Sci. Technol. Int. J. ,20: 1211-1219.

30. Awati V. B., Jyoti M. and Bujurke N. M. 2018. Series Solution of Steady Viscous Flow Between Two Porous Disks with Stretching Motion. Journal Nanofluids, 7: 982-994. 